\title{
RF Energy Transfer Channel Models for Sustainable loT
}

Sidharth Kumar, Swades De and Deepak Mishra

The self-archived postprint version of this journal article is available at Linköping University Institutional Repository (DiVA):

http:/ / urn.kb.se/ resolve?urn=urn:nbn:se:liu:diva-150874

N.B.: When citing this work, cite the original publication.

Kumar, S., De, S., Mishra, D., (2018), RF Energy Transfer Channel Models for Sustainable IoT, IEEE Internet of Things J ournal, 5(4), 2817-2828. https:/ / doi.org/ 10.1109/J IOT.2018.2827936

Original publication available at:

https:/ / doi.org/ 10.1109/J IOT.2018.2827936

Copyright: Institute of Electrical and Electronics Engineers (IEEE)

http:// www.ieee.org/index.html

(C) 2018 IEEE. Personal use of this material is permitted. However, permission to reprint/ republish this material for advertising or promotional purposes or for creating new collective works for resale or redistribution to servers or lists, or to reuse any copyrighted component of this work in other works must be obtained from the IEEE. 


\title{
RF Energy Transfer Channel Models for Sustainable IoT
}

\author{
Sidharth Kumar, Swades De, and Deepak Mishra
}

\begin{abstract}
Self-sustainability of wireless nodes in Internet-ofThings applications can be realized with the help of controlled radio frequency energy transfer (RF-ET). However due to significant energy loss in wireless dissipation, there is a need for novel schemes to improve the end-to-end RF-ET efficiency. In this work, first we propose a new channel model for accurately characterizing the harvested DC power at the receiver. This model incorporates the effects of non-line of sight (NLOS) component along with the other factors, such as, radiation pattern of transmit and receive antennas, losses associated with different polarization of transmitting field, and efficiency of power harvester circuit. Accuracy of the model is verified via experimental studies in an anechoic chamber (a controlled environment). Supported by experiments in controlled environment, we also formulate an optimization problem by accounting for the effect of NLOS component to maximize the RF-ET efficiency, which cannot be captured by the Friis formula. To solve this nonconvex problem, we present a computationally-efficient golden section based iterative algorithm. Finally, through extensive RF-ET measurements in different practical field environments we obtain the statistical parameters for Rician fading as well as path loss factor associated with shadow fading model, which also asserts the fact that Rayleigh fading is not well suited for RF-ET due to presence of a strong LOS component.
\end{abstract}

Index Terms-RF energy transfer, channel characterization, experimental analysis, path loss with shadowing, Rician fading, K-factor, golden section method, method of moments

\section{INTRODUCTION AND BACKGROUND}

With the advent of 5G and Internet-of-Things (IoT), there has been an increasing demand for sustainable wireless networks. Perpetual and autonomous operation of the wireless nodes can now be practically realized with energy harvesting technologies, which enable recharging from ambient resources, such as, solar, wind, or ambient radio frequency (RF). However due to uncertainty in availability of ambient energy, ondemand energy transfer (ET) from dedicated RF sources has emerged as a promising solution. Optimum use of RF-ET technology can aid in achieving perpetual operation of large scale IoT deployments.

The electronic devices that use RF energy are operated by DC power, wherein the received RF energy is converted to DC power and stored in a supercapacitor using power harvester circuit [2], [3]. Efficiency of these circuits is generally a nonmonotonic function of the received power [3]. The models

A preliminary version of the work was presented at the Nat. Conf. Commun. (NCC), Chennai, India, Mar. 2017 [1].

S. Kumar and S. De are with the Department of Electrical Engineering and Bharti School of Telecom, IIT Delhi, New Delhi, India (e-mail: sidharth.kumar, swadesd@ee.iitd.ac.in). D. Mishra is with the Department of Electrical Engineering, Linköping University, Sweden. for estimating the received RF power should account for this non-monotonic nature of RF-to-DC conversion.

Further, limited range is one of the major bottlenecks of RFET technology. The main reason for short range of wireless energy transfer compared to wireless information transfer is the large difference in hardware sensitivities $(-10 \mathrm{dBm}$ versus $-60 \mathrm{dBm})$. Hence, more improved systems are needed to make RF-ET feasible in real-life situations. Also, to best utilize the merits of RF-ET technology, there is a need to correctly characterize the received power with the help of accurate energy propagation models.

\section{A. Related Works}

Commonly used deterministic model for RF-ET in literature is the Friis transmission model [3]-[6]. The Friis transmission equation is valid only in free space propagation. But, this situation is rarely expected to happen in real-life IoT sensor networks because of the node's close proximity to the ground, and most of the times shadow fading effects are also present. In [7], several deterministic and statistical propagation models for mobile communication were discussed. However, these methods cannot be readily applied for characterizing the RFET process because they are derived for a certain physical configuration that is suitable for mobile data communications.

In [8], the authors considered non-line of sight (NLOS) signal component for wireless cellular communication, but their analysis did not account for the antenna radiation pattern and polarization of transmitted field. Moreover, it was assumed that inter-antenna distance is much larger than the antenna height, due to which angle of incidence and reflection coefficient respectively came out to be close to $0^{\circ}$ and -1 . This resulted in a path loss coefficient of 4 . Thus, the model in [8] is not suitable for short range RF-ET.

Some authors have also considered the Rayleigh fading model to characterize the randomness in received power [9]. The problem with statistical models like Rayleigh channel fading is that it gives equal importance to all the components which is not true in RF-ET, because its range is very limited and line-of-sight (LOS) component is always present. Intuitively, Rician fading model is more suited for this purpose. The works in [10], [11] considered Rician fading model for analyzing various aspects of relay assisted RF-ET; however the Rician parameter considered was not experimentally verified.

In [12], the authors conducted experiments in macrocell environments over $1.9 \mathrm{GHz}$ channels and came up with a model for Rician fading parameter $K$ as encountered by fixed end users. The experimental set-up was designed for cellular 
wireless communications, which is not applicable for short range RF-ET. Similarly, the experimental path loss model proposed in [13] is not feasible for RF-ET.

\section{B. Motivation and Research Gap}

Recently, there has been significant interest towards Wireless Powered Communication Network [14], [15] and Simultaneous Wireless Information and Power Transfer [16]. To ensure good performance in these communication paradigms both empirical and statistical channel models are needed which are specifically targeted for short-range RF-ET. In short-range RF-ET experiments with varied position of shadowing and obstructing elements, reflection gain was observed in [17], which is contrary to the general observation with 2-ray propagation model in long-range signal communication. This interesting observation motivates us to characterize the RF-ET gain in presence of multipath components. It may be noted that, the reflected waveform acts as an NLOS component, which can have magnitude comparable to that of the LOS component when the path difference is not large - as in RF-ET scenario.

We note that the analytical models and experimental observations on signal propagation characteristics for wireless communications are not applicable in short-range RF-ET. Moreover, the experimental findings on Rician $K$-factor in cellular wireless environments do not apply in RF-ET. Although there are some limited theoretical studies on RFET that consider Rician channel [10], [11], no experimental study has been reported in the literature that characterizes the Rician parameter $K$ along with path loss factor and shadow fading parameter for various IoT communication scenarios. Thus, there is a clear lacuna on analytical modeling and experimental characterization of $R F$ energy propagation and analysis of stochastic nature of wireless channel for RF-ET.

\section{Contributions and Significance}

In this work, we investigate the effect of NLOS components in RF-ET performance. A deterministic receive power model is developed and validated by hardware experiments in controlled environment. The developed model allows to optimize the RF-ET gain by optimal positioning of the RF source and the receiver. Further, a statistical channel model is proposed that captures the strong LOS energy component, and the parameters are estimated by practical field experiments. Highlights of contributions of this work are the following:

1) A new deterministic channel model is proposed for RFET characterization by incorporating the practical factors of electromagnetic wave propagation (Sections III, IIIT).

2) The proposed model is validated experimentally in controlled environment (Section IV).

3) An optimization formulation is presented to capture how NLOS component can cooperate with the LOS component in providing significant RF-ET gain (Section $\sqrt{V}$.

4) To capture the strong LOS component in statistical channel variation in practical field settings, the RF-ET channel is modeled as Rician channel (Section VI).

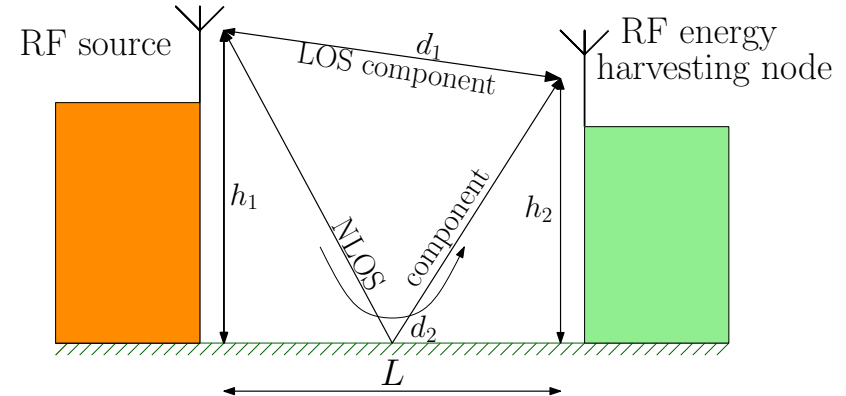

Fig. 1: Proposed channel model for incorporating the effect of NLOS component in RF energy transfer.

5) Numerical results capture the optimum conditions for maximizing RF-ET gain, and the fading channel parameters in various typical field deployment scenarios are estimated via extensive RF-ET experiments (Section VIIT).

To the best of our knowledge, this is the first experimentallydriven work that characterizes the RF energy signal propagation (both deterministic and statistical), specifically targeted for wireless RF-ET in real-life scenarios.

The proposed model can be applied in various practical RF-ET scenarios where other reflectors are present besides ground. For the cases where deterministic model makes the analysis complex, the statistical model of either Rician fading or path loss with shadow fading can be employed to arrive at interpretable results. The other possible applications include design of efficient harvester circuits by optimizing the received power, and medium access control and routing protocols to effectively divide the time between information and energy transfer to maximize the network lifetime.

\section{Problem Definition}

One objective of this work is to develop a practical path loss model for RF-ET by incorporating the NLOS component due to ground reflection, which cannot be captured by the free space propagation model. We characterize the received power at the RF energy harvesting destination as a function of its distance from the RF source, heights at which the source and destination nodes are placed, and the other RF parameters, such as, polarization, radiation pattern, and matching losses. In this section, first we discuss the conventional RF-ET model that is based on Friis propagation model. After that we provide outline of the proposed channel model which addresses the drawbacks of the conventional model.

As a general rule of thumb all unit vectors are represented with an overhead hat (e.g. $\hat{u}$ ) whereas all other vectors are represented with an overhead arrow (e.g. $\vec{u}$ ). All other considered variables are scalar quantities.

\section{A. Conventional RF Energy Transfer Model}

In our considered system set-up (depicted in Fig. 1), the source node transmits energy to the destination (or end) node via RF energy radiation. The transmitter and the energy harvesting receiver are respectively at heights $h_{1}$ and $h_{2}$ from the ground, and are separated by a horizontal distance $L$. The source node consists of a processor, crystal oscillator, 
a directional antenna, and sufficient energy resource (e.g., a battery or power grid connection) to perform its operations. In field applications, the source could be mounted on a robot/vehicle which moves around and charges the end nodes as per a schedule. Since the wireless charging is a timeconsuming process the source remains static until the end node is charged. The energy harvesting node consists of a rechargeable battery or supercapacitor which is charged by the RF energy harvester circuit. The receiver antenna, which is omnidirectional, captures the electromagnetic energy and supplies to the rectification circuit. Friis transmission equation which is based on free space model, is generally used to calculate the received power [3]-[6]. The Friis model gives received power $P_{r}^{\text {(Friis) }}$ as inverse square law, as per the following equation [18]:

$$
\frac{P_{r}^{(\text {Friis })}}{P_{t}}=G_{t} G_{r}\left(1-\left|\Gamma_{t}\right|^{2}\right)\left(1-\left|\Gamma_{r}\right|^{2}\right)\left(\frac{\lambda}{4 \pi d_{1}}\right)^{2}\left|\hat{e}_{n} \cdot \hat{e}_{r}\right|^{2} .
$$

Here $P_{r}^{(\text {Friis })}$ is a function of transmit power $P_{t}$, distance $d_{1}$, wavelength $\lambda$ of the electromagnetic wave, antenna gains $G_{t}, G_{r}$, and circuit reflection coefficients $\Gamma_{t}, \Gamma_{r}$ of the antennas along with the corresponding polarization loss factor $\left|\hat{e}_{n} \cdot \hat{e}_{r}\right|^{2}$.

\section{B. Proposed Channel Model for RF Energy Transfer}

RF energy propagates through wireless medium in the form of electric and magnetic fields which are transmitted and received with the help of antennas. The typical distances in RF-ET are in the far field of antennas, where far field distance is approximately given by $d_{f}=\frac{2 D^{2}}{\lambda}$. Here $D$ is the maximum linear dimension of the antenna. In far field of antennas the emanating electromagnetic waves behave like planar waves, where the amplitude decreases inversely with distance $d_{1}$. For a monochromatic planar wave emanating from an antenna, the electric field $\vec{E}\left(d_{1}, t\right)$ at a distance $d_{1}$ can be written as

$$
\vec{E}\left(d_{1}, t\right)=\frac{E_{o} d_{o}}{d_{1}} e^{i\left(k \cdot d_{1}-2 \pi f t\right)} \hat{n} .
$$

Here $d_{o}$ is some representative distance at which the magnitude of electric field at time $t$ is $E_{o}$, and it can be used to calculate the magnitude of electric field $\vec{E}$ at any other distance. The symbol $k=\frac{2 \pi}{\lambda}$ used in above equation is wave number of the wave. After an RF wave emanates from an antenna it propagates in certain directions, controlled by the antenna gain. This wave can reach the receiving antenna either directly with magnitude $\left|\vec{E}_{L O S}\right|$, or after reflection. The most obvious reflector is ground. The reflected wave also reaches the receiving antenna but with a different magnitude $\left|\vec{E}_{L N O S}\right|$. This magnitude can be quite different from the $\left|\vec{E}_{L O S}\right|$, as it depends on the antenna gain in that direction, polarization of the transmitted field, reflection coefficient of the ground, and the path it travels before reaching the target. The total electric field $\vec{E}_{\text {Total }}$ at the energy harvesting receiver is:

$$
\vec{E}_{\text {Total }}=\vec{E}_{L O S}+\vec{E}_{N L O S} .
$$

This total electric field in 3 is used next for computing the harvested RF power.

\section{Proposed Received Power Equation}

In this section we derive the expression of total power at the receiver by incorporating the effect of NLOS component. With $\eta$ as the intrinsic impedance of free space, the received power $P_{r}$ at the antenna due to the impinged electric field $\vec{E}_{\text {Total }}$ and an effective vector length $\vec{L}$ is given by:

$$
P_{r}=\left(\vec{E}_{\text {Total }} \cdot \vec{L}\right)^{2} / \eta \text {. }
$$

\section{A. Effect of NLOS Component}

The main effect of NLOS component is that it changes the effective electric field at the receiver, which is given by (3). The dominant effect of NLOS component is because of its path difference $\Delta d$ with the LOS component. $\Delta d$ leads to a phase difference $\Delta \phi=k \cdot \Delta d$, which can make the two components add constructively or destructively. Since the NLOS component incurs additional losses due to ground reflection and extra path length, the received signal is not completely decimated due to destructive interference with the LOS component.

\section{B. Total Mean Harvested Power at RF Harvesting Destination}

The electric field in (2) at a distance $d_{1}$ $\left(=\sqrt{L^{2}+\left(h_{1}-h_{2}\right)^{2}}\right)$ from the transmit antenna (cf. Fig. 1] and in unit direction $\hat{e}_{r 1}$ can be written as [18]:

$$
\vec{E}=\sqrt{\left(1-\Gamma_{t}^{2}\right) \frac{\eta P_{t} G_{t}\left(\theta_{1}^{t}, \phi_{1}^{t}\right)}{4 \pi d_{1}^{2}}} e^{-j k d_{1}} \hat{e}_{r 1} .
$$

Here $P_{t}$ is the transmit power, $G_{t}$ is the gain of transmit antenna in radial direction $(\theta, \phi), \eta$ is the intrinsic impedance of free space, and $\Gamma_{t}$ is the reflection coefficient due to impedance mismatch between antenna and transmission line which is given by $\Gamma_{t}=\frac{Z_{i}-Z_{o}}{Z_{i}+Z_{o}}$ with $Z_{i}$ as the input impedance of the antenna and $Z_{o}$ as the characteristic impedance of the transmission line. After reflection the electric field changes to:

$$
\vec{E}_{2}=\Gamma\left(\theta_{i}\right) \sqrt{\left(1-\Gamma_{t}^{2}\right) \frac{\eta P_{t} G_{t}\left(\theta_{2}^{t}, \phi_{2}^{t}\right)}{4 \pi d_{2}^{2}}} e^{-j k d_{2}} \hat{e}_{r 2} .
$$

Here $d_{2}\left(=\sqrt{h_{1}^{2}+\left(\frac{h_{1} L}{h_{2}+h_{1}}\right)^{2}}+\sqrt{h_{2}^{2}+\left(\frac{h_{2} L}{h_{2}+h_{1}}\right)^{2}}\right)$ is different from $d_{1}$ in (5), as the reflected field traverses a different path length. $\Gamma\left(\theta_{i}\right)$ is the reflection coefficient of the ground of relative permitivity $\epsilon_{r}$, which is different for each polarization and angle of incidence $\theta_{i}$, given by [19]:

$$
\begin{gathered}
\Gamma_{\perp}\left(\theta_{i}\right)=\frac{\sin \theta_{i}-\sqrt{\epsilon_{r}-\cos ^{2} \theta_{i}}}{\sin \theta_{i}+\sqrt{\epsilon_{r}-\cos ^{2} \theta_{i}}} \quad: \text { Horizontal polarization } \\
\Gamma_{\|}\left(\theta_{i}\right)=\frac{-\epsilon_{r} \sin \theta_{i}+\sqrt{\epsilon_{r}-\cos ^{2} \theta_{i}}}{\epsilon_{r} \sin \theta_{i}+\sqrt{\epsilon_{r}-\cos ^{2} \theta_{i}}} \quad: \text { Vertical polarization. }
\end{gathered}
$$

Based on polarization of the antenna used, the electric field will have different polarization for which respective reflection coefficient has to be used. The effective vector length $\vec{L}$ of the receiving antenna is given by:

$$
\vec{L}=\left(\sqrt{\left(1-\Gamma_{r}^{2}\right) \frac{\lambda^{2}}{4 \pi} G_{r}\left(\theta^{r}, \phi^{r}\right)}\right) \hat{e}_{n} .
$$




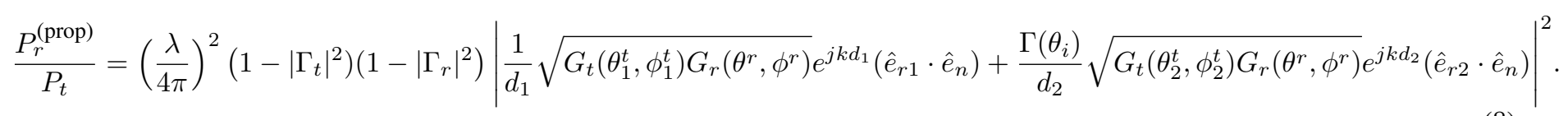

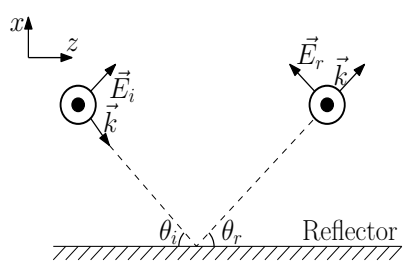

(a) Vertical Polarization

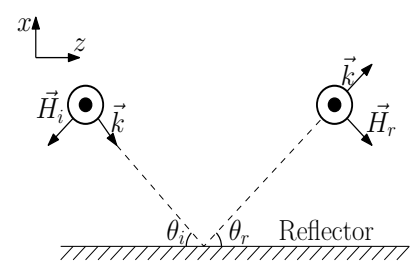

(b) Horizontal Polarization

Fig. 2: The reflected and incident field directions for different polarizations, here dot represents field coming out of plane.

The effective vector length points into the unit direction $\hat{e}_{n}$ in which receiving antenna is polarized. Received power depends on the dot product of received field and vector effective length, and it is incorporated to include the effects of mismatch in between the polarization of radiated field $\hat{e}_{r}$ and the receiving antenna $\hat{e}_{n}$. Therefore, the power received depends on (3), which is in turn obtained by adding $E_{L O S}$ and $E_{N L O S}$. The values of $E_{L O S}$ and $E_{N L O S}$ are respectively given by (5) and (6). Hence, by substituting all the quantities into (4), the proposed estimate of total received power $P_{r}^{\text {(prop) }}$ is obtained in (8). This equation is general where $\Gamma\left(\theta_{i}\right)$ is dependent on the polarization of the transmitted RF signal.

The main motivation behind considering only two rays here is that the reflecting surface is smooth, and according to geometrical optics the angle of incidence is equal to angle of reflection, hence only one reflected ray will reach the destination [19]. Also most of the real-life situations where RF energy transfer is used, we have smooth ground plane.

\section{Curious Case of Vertical Polarization}

The polarization loss factor terms in (8) evaluate to ' 1 ' for the case of horizontal polarization, but need to be specifically evaluated for vertical polarization. To understand this, see the pictorial view in Fig. 2. With horizontal polarization, the $\vec{E}$ field is perpendicular to the $x-z$ plane and remains so even after reflection, which is due to the fact that for plane waves the electric field, magnetic field, and the propagation vector follow the relation $\hat{B}=\hat{k} \times \hat{E}$ and boundary conditions during reflection [20, ch.9]. For vertical polarization $\vec{H}$ is out of plane and remains so after reflection, but the electric field changes direction and hence the polarization loss factors become:

$$
\begin{aligned}
& \hat{e}_{r 1} \cdot \hat{e}_{n}=\cos \left(\theta_{1}\right)=\frac{L}{\sqrt{L^{2}+\left(h_{2}-h_{1}\right)^{2}}} \\
& \hat{e}_{r 2} \cdot \hat{e}_{n}=\cos \left(\theta_{2}\right)=\frac{L}{\sqrt{L^{2}+\left(h_{2}+h_{1}\right)^{2}}}
\end{aligned}
$$

The effects of electric field polarization on the received power level as a function of transmitter and receiver node positions will be considered in experimental validation of

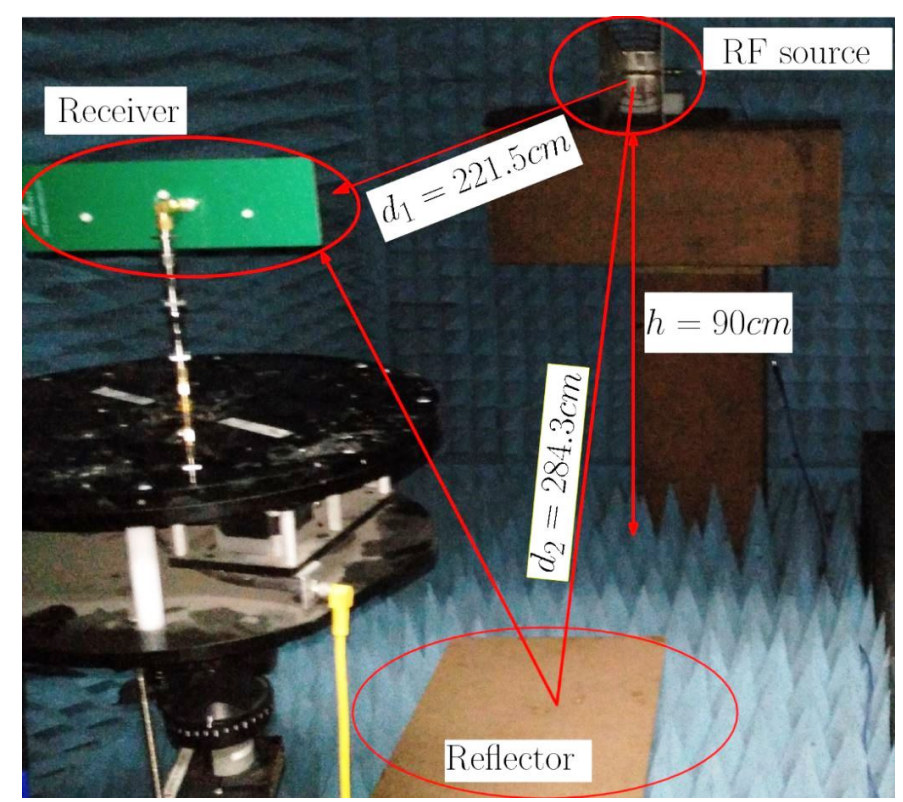

Fig. 3: Experimental set-up in the anechoic chamber.

the proposed received RF power estimation (Section IV), for performance optimization with the proposed deterministic channel model (Section $\mathrm{V}$ ), and further in statistical channel parameter estimation (Section VII).

\section{EXPERIMENTAL VALIDATION}

In this section, we present validation of the estimated received power expression given by (8) through RF energy transfer experiments.

\section{A. Experimental Set-up}

Experimental set-up in the anechoic chamber (Fig. 3 comprised of the following units:

- $\boldsymbol{R} F$ source: A Hittite RF synthesizer HMC-T2100 was used as RF source. It was set to transmit at a fixed frequency of $915 \mathrm{MHz}$ and fixed transmit power of 0 $\mathrm{dBm}$.

- Spectrum analyzer: An Anritsu Spectrum Analyzer MS2719B was used to analyze the frequency and power profile of the received power.

- Data acquisition and hardware control: The data related to received power and the rotation of receiving antenna using stepper motor is fully software controlled with the help of DAMS Antenna Measurement Studio. The acquired data was stored in a text file.

- Transmit antenna: An ETS.LINDGREN double ridged waveguide horn (model number 3115) was used as the transmit antenna which was fed through RF synthesizer. 
TABLE I: Experimental parameter values.

\begin{tabular}{|l|l||l|l|}
\hline Parameter & Value & Parameter & Value \\
\hline \hline$P_{t}$ & $0 \mathrm{dBm}$ & $\Gamma_{\|}\left(\theta_{i}\right)_{\text {cardboard }}$ & -0.0323 \\
\hline $\mathrm{h}$ & $90 \mathrm{~cm}$ & $\Gamma_{\perp}\left(\theta_{i}\right)_{\text {cardboard }}$ & -0.3043 \\
\hline$d_{1}$ & $221.5 \mathrm{~cm}$ & $\Gamma_{\|}\left(\theta_{i}\right)_{\text {metal }}$ & 1 \\
\hline$d_{2}$ & $284.3 \mathrm{~cm}$ & $\Gamma_{\perp}\left(\theta_{i}\right)_{\text {metal }}$ & -1 \\
\hline$\theta_{i}$ & $39.29^{\circ}$ & $G_{\text {dir }}$ & 4.1 \\
\hline$\left(\Gamma_{t}\right)_{\text {omni }}$ & 0.8462 & $G_{\text {omni }}$ & 1.25 \\
\hline$\left(\Gamma_{t}\right)_{\text {dir }}$ & 0.8117 & $\lambda$ (wavelength) & $0.3278 \mathrm{~m}$ \\
\hline
\end{tabular}

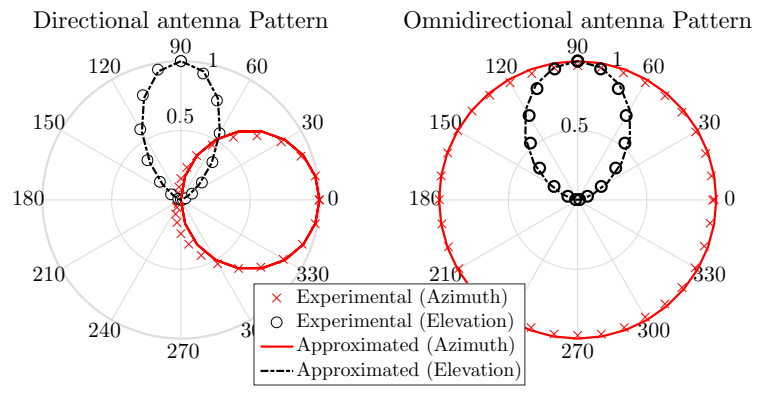

Fig. 4: Normalized antenna radiation patterns.

- Receive antenna: The experiment was conducted for two types of receive antennas, both available from Powercast [2]. These are the most commonly used antennas for RFET. The first antenna is directional with linear gain 4.1 $(6.1 \mathrm{dBi})$ and half power beamwidth of $122^{\circ}$ (azimuth) and $68^{\circ}$ (elevation). The other antenna is omnidirectional in azimuth plane with linear gain $1.25(1.0 \mathrm{dBi})$.

- Reflectors: To emulate the reflector $\Gamma_{t}$ behavior in anechoic chamber we used copper metal sheet $\left(\epsilon_{r}=\infty\right)$ and cardboard $\left(\epsilon_{r}=2\right)$.

\section{B. Empirical Parameters}

The experimental parameters are given in Table I

For empirical verification of the receiver power estimate in (8), radiation patterns of the antennas are needed. Radiation pattern of the horn antenna is available in [21]. To characterize the radiation pattern of Powercast antennas, the analytical expressions (11) are proposed to closely match the empirical readings, as shown in Fig. 4. The azimuth (horizontal) plane is traversed by $\phi \in[0,2 \pi]$, whereas the elevation plane is traversed by $\theta \in[0, \pi]$ variation.

$$
G(\theta, \phi)= \begin{cases}G_{d i r} \cos (\phi) \sin ^{4}(\theta) & : \text { Directional } \\ G_{\text {omni }} \sin ^{2}(\theta) & : \text { Omnidirectional. }\end{cases}
$$

\section{Experimental Results and Observations}

To verify the accuracy of the derived analytical expression in (8), the received power values obtained experimentally and from (8) are plotted in Fig. 6. To study the effects of polarization, the experiments were conducted for both horizontal and vertical polarizations. The different polarizations are realized by rotating antennas as shown in the Fig. 5 .

For measuring the received power with varying azimuth and elevation angles, the RF source and directional antenna were placed respectively with horizontal and vertical polarization.

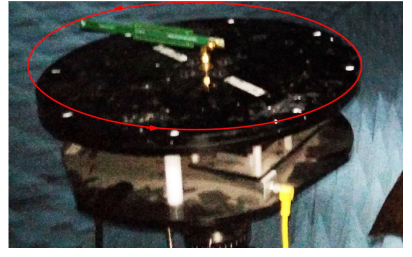

(a) Elevation plane

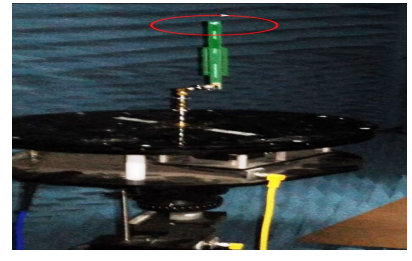

(b) Azimuth (horizontal) plane
Fig. 5: The sense of rotation of antenna for different polarization.

The corresponding received power values are plotted in Figs. 6.a) and 6(b). In Fig. 6(c), power received with omnidirectional antenna and vertical polarization is plotted. Root mean square errors (RMSE) of the analytically obtained values with respect to the experimental data in these three different set-ups are listed in Table II] which are within the acceptable upper limit of 0.08 [22] - thus validating the developed analytical expression in 8 .

TABLE II: RMSE of the analytically computed values with respect to the experimental data.

\begin{tabular}{|c|c|c|c|}
\hline \multirow[b]{2}{*}{ Set-up } & \multicolumn{3}{|c|}{ RMSE } \\
\hline & Fig. 6 a) & Fig. 6 (b) & Fig. 6 c) \\
\hline No reflector & 0.0583 & 0.0385 & 0.0639 \\
\hline Metal & 0.0599 & 0.0750 & 0.0501 \\
\hline Cardboard & 0.0587 & 0.0335 & 0.0597 \\
\hline
\end{tabular}

With this validation of the received power estimate, we next aim to maximize the energy transfer efficiency by optimally placing the RF transmitter and the harvester node for a given RF-ET hardware parameter values.

\section{MAXIMIZING RF ENERGY TRANSFER EFFICIENCY}

We now aim to maximize the RF-ET efficiency as a function of RF source and harvester node placement, which optimizes the combined effect of LOS and NLOS received energy components. Since in our experimental set-up the source-receiver distance cannot be changed, we propose to adjust the source and receiver heights from the ground plane. In particular, we propose a partitioned range golden search algorithm to maximize the end-to-end RF-ET efficiency.

This optimization study is motivated by the fact that, a practical set-up for wireless charging of end nodes involves an RF energy mule that goes from node to node to supply energy via RF radiation, and it also collects the data along the way [14]. The mule tries to move as close as possible to the end node for fast energy transfer. However, due to terrain conditions, or physical obstacles, or strategic reasons, it may not be possible for the mule to place itself beyond a close proximity to the field node. In such a situation, for a given source-to-receiver distance, the energy mule can adjust its antenna height to maximize the RF-ET rate.

\section{A. Optimization Problem Formulation}

In Section III we have derived the received power (8) as a function of source-to-receiver distance and the source and receiver antenna heights above ground. Due to interaction 


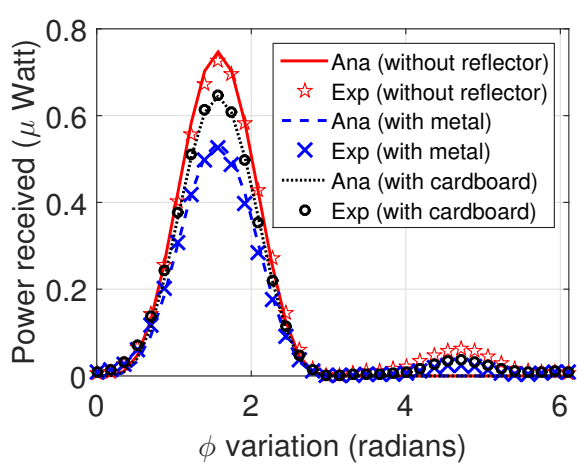

(a) Directional; horizontal polarization

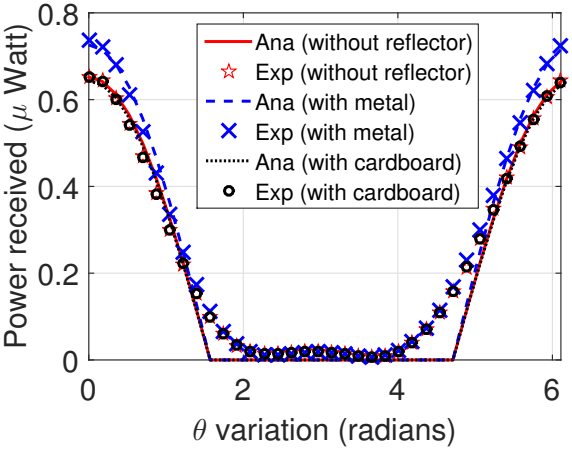

(b) Directional; vertical polarization

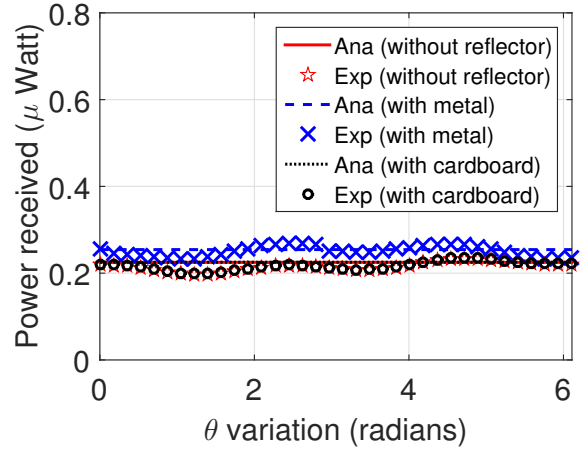

(c) Omnidirectional; vertical polarization

Fig. 6: Power received at different types of antennas with different polarizations.

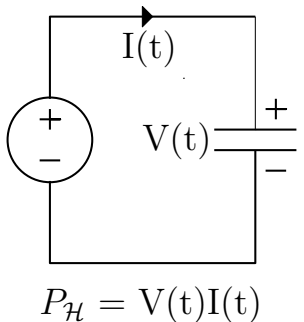

(a)

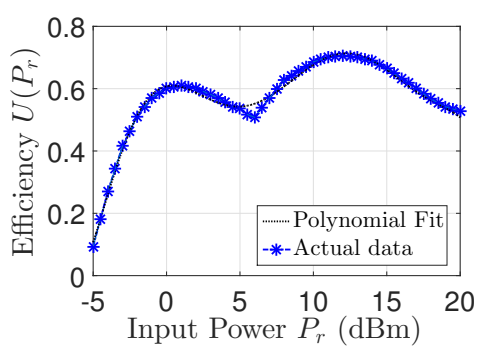

(b)
Fig. 7: (a) Supercapacitor model for constant power charging. (b) RF-to-DC conversion efficiency of Powercast P1110 RF energy harvester and its comparison with the fitted model.

of the LOS and NLOS wavefronts, for a given fixed internode distance and the receiver antenna height $h_{2}$, there is an optimum source antenna height $h_{1} \in\left[h_{\min }, h_{\max }\right]$ at which power received is maximum. Further, it is known that, energy harvesting efficiency $U$ of the harvesting circuit is not a monotonic function of received power [2]. The RFto-DC conversion efficiency $U$ of the Powercast P1110 IC as a function of received power $P_{r}$ is plotted in Fig. 7 (b). We approximate this function by a 7-degree polynomial [23], which is expressed as:

$$
\begin{aligned}
& U\left(P_{r}\right)=\left(-2.711 \times 10^{-8}\right) P_{r}^{7}+\left(1.566 \times 10^{-6}\right) P_{r}^{6} \\
& -\left(2.858 \times 10^{-5}\right) P_{r}^{5}+\left(9.445 \times 10^{-5}\right) P_{r}^{4} \\
& +\left(2.046 \times 10^{-3}\right) P_{r}^{3}-(0.01465) P_{r}^{2}+(0.01064) P_{r}+0.6077 .
\end{aligned}
$$

R-squared and RMSE of the polynomial fit are 0.9949 and 0.0125 respectively, which are within the allowable limit [22].

The end-to-end RF-ET efficiency $\zeta$ is defined as the ratio of DC power harvested to the RF power transmitted. For a constant transmitted power $\left(P_{t}\right)$, inter-node distance $L$, and fixed receiver height $h_{2}$, there exists an optimum height $h_{\text {opt }}$ of transmitter for which maximum RF-ET efficiency $\zeta_{o p t}$ is achieved. Hence, the following relationship holds:

$$
\begin{gathered}
\zeta_{o p t}^{(\text {prop })}=\frac{P_{\mathcal{H}}^{(\text {prop })}\left(h_{\text {opt }}\right)}{P_{t}}=\frac{P_{r}^{(\text {prop })}\left(h_{o p t}\right) \cdot U\left(P_{r}^{(\text {prop })}\left(h_{o p t}\right)\right)}{P_{t}} \\
>\frac{P_{\mathcal{H}}^{(\text {Friis })}\left(h_{\text {opt }}\right)}{P_{t}}=\frac{P_{r}^{(\text {Friis })}\left(h_{o p t}\right) \cdot U\left(P_{r}^{(\text {Friii })}\left(h_{o p t}\right)\right)}{P_{t}}=\zeta_{\text {opt }}^{(\text {Friis })}
\end{gathered}
$$

where $P_{\mathcal{H}}^{(\text {prop })}\left(h_{\text {opt }}\right)$ is the optimum harvested power obtained by setting $h_{1}=h_{\text {opt }}$ in the proposed received power expression in (8) and incorporating $P_{r}=P_{r}^{(\text {prop })}\left(h_{\text {opt }}\right)$ in (12). Similarly, $P_{\mathcal{H}}^{\text {(Friis) }}\left(h_{\text {opt }}\right)$ is the harvested power obtained by setting $h_{1}=h_{o p t}$ in (1) and putting $P_{r}=P_{r}^{(\text {Friis })}\left(h_{\text {opt }}\right)$ in (12). One notable observation here is that, the proposed channel model can result in a better RF-ET efficiency than that with the Friis model. This can be achieved when proper care is taken while placing the transmitter antenna. The optimal placement must be such that the LOS and NLOS components add up constructively at the receiver, thereby transferring the maximum energy. Hence, our objective is to find the optimum height $h_{\text {opt }}$ which offers the maximum end-to-end RF-ET efficiency, i.e., the maximum $P_{\mathcal{H}}$. Accordingly, the optimization problem $(\mathcal{P})$ is defined as:

$$
\begin{array}{lll}
(\mathcal{P}): & \underset{h}{\operatorname{maximize}} \quad \zeta=\frac{P_{\mathcal{H}}\left(h_{1}\right)}{P_{t}} \\
& \text { subject to: } \quad h_{\min } \leq h_{1} \leq h_{\max } .
\end{array}
$$

\section{B. Harvested Power $P_{\mathcal{H}}$ Variation with Transmitter Height}

To solve the optimization problem (14), we first study the received power variation as a function of antenna height. For this purpose, the RF source/transmitter (energy mule) has antenna gain $6.1 \mathrm{dBi}$ and the receiver (field node) has antenna gain $1.0 \mathrm{dBi}$. The other hardware parameters are considered the same as described in Section IV-A To study the effect of polarization, both horizontal and vertical polarization are considered. To numerically compute the variation of harvested power $P_{\mathcal{H}}$ with the changes in the transmitter height, four different scenarios are considered, as mentioned in Table III]

TABLE III: System parameters for different scenarios.

\begin{tabular}{|c|c|c|c|}
\hline Scenario & $L(\mathrm{~m})$ & $h_{2}(\mathrm{~m})$ & $h_{1}\left(h_{\min }, h_{\max }(\mathrm{m})\right)$ \\
\hline I & 0.8 & 1 & $(0.15,1.5)$ \\
\hline II & 1.8 & 1 & $(0.15,1.5)$ \\
\hline III & 0.8 & 0.15 & $(0.15,1.5)$ \\
\hline IV & 2 & 2 & $(0.15,1.5)$ \\
\hline
\end{tabular}

Harvested power variation as a function of transmitter antenna height for both polarizations are plotted in Figs. 8(a)-(d) for the above-mentioned four scenarios. The main observation is that, the harvested power $P_{\mathcal{H}}$ as a function of transmit 

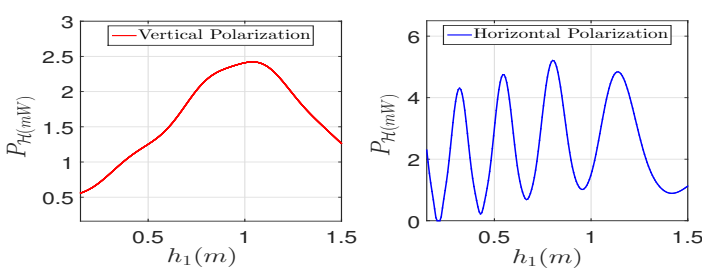

(a)
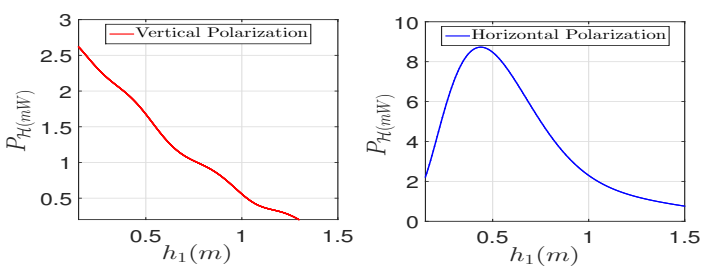

(c)
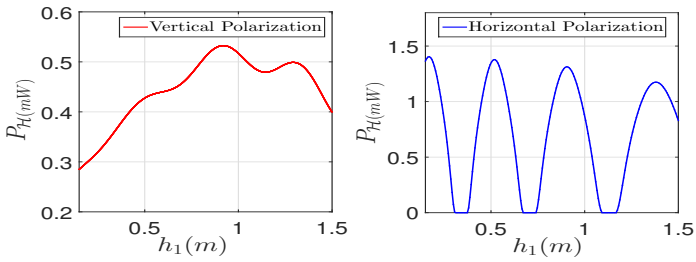

(b)
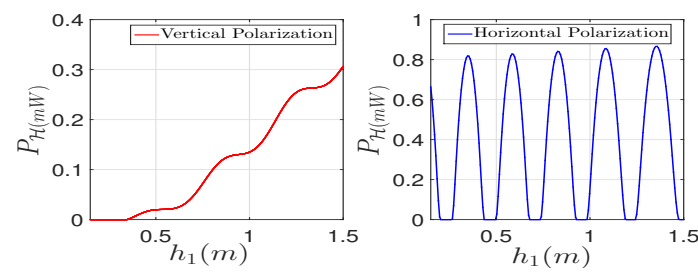

(d)

Fig. 8: Harvested power variation as a function of transmitter antenna height in the four scenarios with both polarizations: (a) Scenario I, (b) Scenario II, (c) Scenario III, and (d) Scenario IV.

antenna height $h_{1}$ is highly non-convex in nature. Also, $P_{\mathcal{H}}$ shows a strong dependence on the signal polarization. Specifically more oscillatory behavior of $P_{\mathcal{H}}$ is observed for horizontally polarized signal, because the polarization loss factor for vertical polarization is very less than that for horizontal polarization as explained in Section III-C. The performance varies for all the different scenarios. For example, Scenario III shows less oscillations as compared to the other cases, the main reason being less height of the receiver antenna. These behaviors also clearly show the importance of optimum antenna heights for maximized $P_{\mathcal{H}}$. In the following, we consider numerical nonconvex optimization method specific to the problem at hand.

\section{Proposed Optimization Algorithm}

We observe that, although the harvested power $P_{\mathcal{H}}$ and hence $\zeta$ is a nonconvex function of $h_{1}, P_{\mathcal{H}}$ is a piece-wise unimodal function of $h_{1}$ (cf. (8) and Fig. 8). Therefore, we first divide the feasible region into equal partitions and then apply golden search algorithm on each partition. Finally, we take the maximum of power harvested from each of these partitions. The golden search algorithm works by reducing the current interval size by a fixed ratio of 0.618 at the end of each iteration, till the interval reduces to a small acceptable level. This algorithm gives correct answers only for those functions which are proven to be unimodal over the required domain. Hence, the whole domain of solution space need to be partitioned in such a way that the objective function remains unimodal within the individual partition windows. Following partitioning of the received power variation, golden section method can be applied over each partition. The steps involved are detailed in Algorithm 1, where the counter returns the total number of computations.

\section{Complexity Analysis}

To investigate performance and complexity of the proposed algorithm to solve the optimization problem, brute

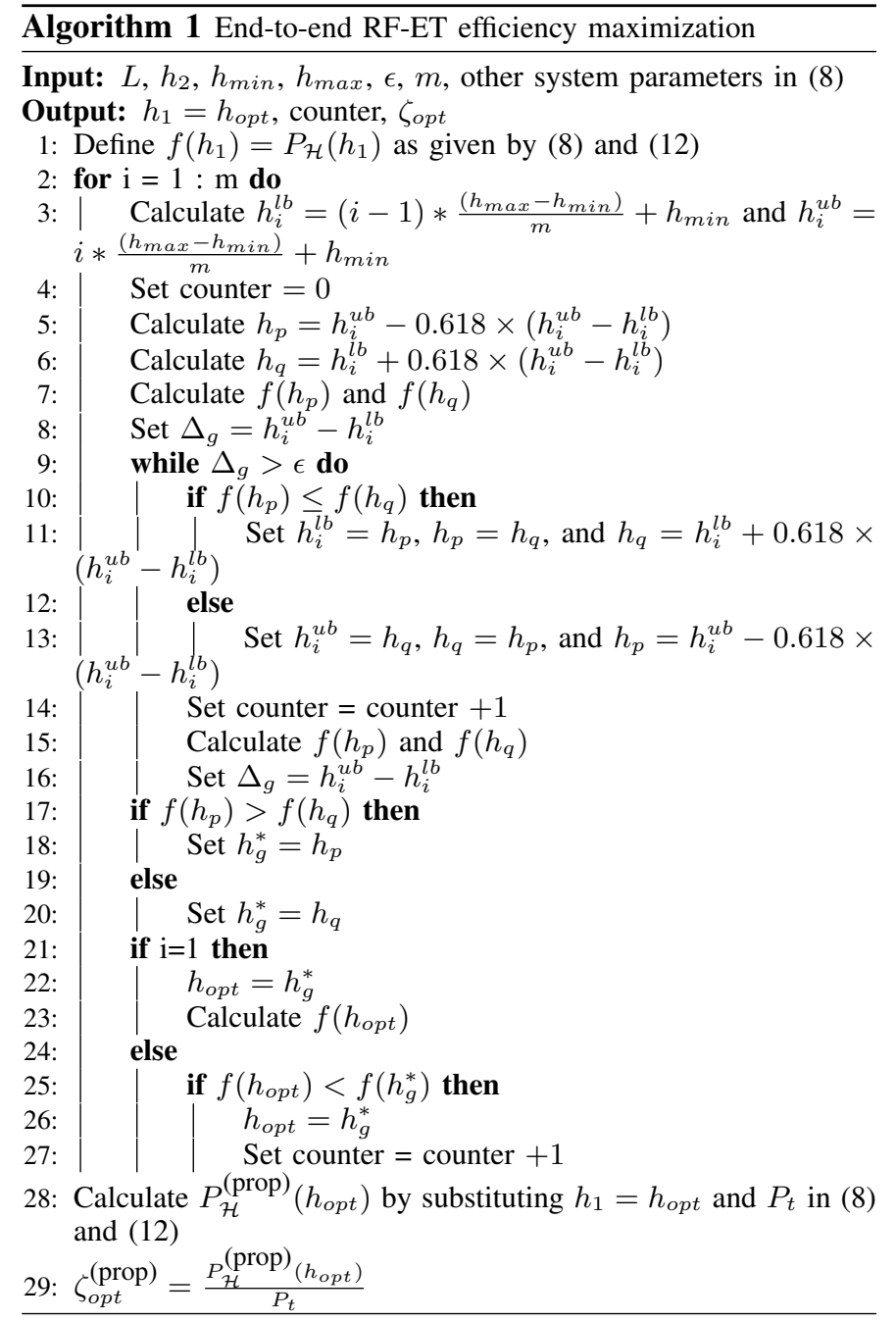

force method is taken as the benchmark. In this method the total number of computations are $2 n-1$, where $n=$ $1+\frac{h_{\max }-h_{\min }}{\epsilon}$ and $\epsilon$ is an acceptable tolerance. For golden section method it is known that the number of computations is 
$\left[2+\frac{\ln \left(\frac{\epsilon}{h_{\max }-h_{\min }}\right)}{\ln (0.618)}\right]$. However, in the proposed algorithm, we apply golden search repeatedly for each partition. Denoting the number of partitions as $m$, the total number of computations required for function evaluation and comparison is:

$$
=m-1+m\left\lceil 2+\frac{\ln \left(\frac{m \cdot \epsilon}{h_{\max }-h_{\min }}\right)}{\ln (0.618)}\right\rceil \text {. }
$$

From 15 it can be inferred that the proposed algorithm has logarithmic complexity in comparison with the brute force method when evaluated as a function of $\epsilon$.

Complexity-accuracy tradeoff and the numerical results on optimum RF-ET performance are presented in Section VII-A.

\section{Statistical Channel Parameter Estimation}

Even though we have proposed evaluated an accurate model to calculate the power received in RF-ET in Section II. this model is reasonably valid in controlled environments such as in a laboratory settings. However, when all surfaces, blockages, and dynamic scattering effects are accounted - as in practical field deployments, a stochastic receive power model is expected to be more reasonable. Since RF-ET distance is much smaller compared to the conventional wireless information communication range, the statistical RF-ET channel model can be better modeled as a Rician channel.

In this section we first present the Rician fading model and then characterize the path loss and shadowing parameters to complete the stochastic modeling. Later, we describe the experimental set-up to find the statistical channel parameters.

\section{A. Rician Parameter Characterization}

In Rician fading channel, received power is distributed according to the non-central $\chi^{2}$ distribution with PDF (probability density function) of received power given by [24, eq. (2.16)]:

$$
f_{P_{r}}(x, K, \Omega)=\frac{(1+K) e^{-K}}{\Omega \exp \left[\frac{1+K}{\Omega} x\right]} I_{o}\left(2 \sqrt{\frac{K(K+1) x}{\Omega}}\right) .
$$

Here $K$ is the Rice factor which represents the ratio of power in main (LOS) component and the sum of all other weaker (NLOS) components, $\Omega$ is the average power combined, and $I_{o}(\cdot)$ is the modified zeroth order Bessel function of first kind. The distribution defined in 16 have two degrees of freedom, namely, Rician factor $K$ and average power $\Omega$. Both of them can be evaluated from the experimental data by using the method of moments [25]. Accordingly, the complex path gain $g(t)$ consisting of a constant term $V$ and other zero mean random time-varying term $v(t)$ can be expressed as:

$$
g(t)=V+v(t)
$$

The two moments of interest are the mean $\Omega$ and RMS fluctuation $G_{r m s}$ around this mean. Defining $\sigma^{2} \equiv \overline{|v(t)|^{2}}$, it has been shown in [25] that:

$$
\begin{gathered}
\Omega=|V|^{2}+\sigma^{2}, \\
G_{r m s}=\left[\sigma^{4}+2|V|^{2} \sigma^{2}\right]^{\frac{1}{2}} .
\end{gathered}
$$

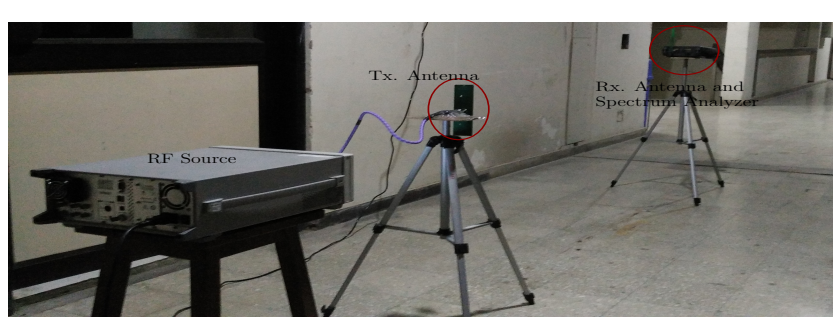

Fig. 9: Set-up for factory floor scenario.

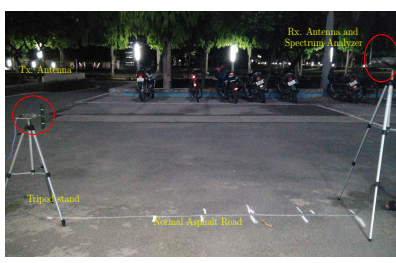

(a) Roadside

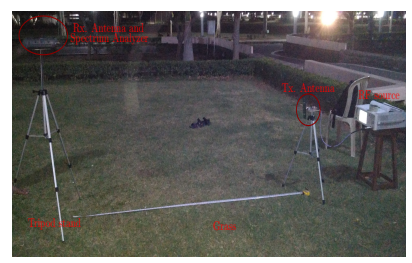

(b) Garden
Fig. 10: Other practical set-ups.

Solving these equations we get:

$$
\begin{gathered}
|V|^{2}=\left[\Omega^{2}-G_{r m s}^{2}\right]^{\frac{1}{2}} \\
\sigma^{2}=\Omega-\left[\Omega^{2}-G_{r m s}^{2}\right]^{\frac{1}{2}} .
\end{gathered}
$$

From (20) and 21), $K$-factor is obtained as:

$$
K=\frac{|V|^{2}}{\sigma^{2}}
$$

\section{B. Statistical Path Loss and Shadowing Model}

Path loss along with shadowing is used to incorporate the effect of hindrances caused to the signal by reflection, diffraction, absorption and scattering by the different obstacles. In this model path loss is represented as sum of a distance dependent term and a statistical term representing shadow fading. Beyond a certain distance $z_{o}$, the path loss in decibel at a distance $z$ can be written as [26]:

$$
P L(\text { in } \mathrm{dB})=A+10 \gamma \log _{10}\left(\frac{z}{z_{o}}\right)+s, \quad z_{o} \leq z
$$

where $\gamma$ is the path loss exponent, $A$ is the path loss (dB) at the distance $z_{o}$ and $s$ is a random variable representing lognormal shadow fading with mean $\mu$ and variance $\varsigma$.

\section{Experimental Set-up}

For estimating the Rician parameter $K$, path loss exponent $\gamma$, and shadowing parameters $\mu$ and $\varsigma$ values, we have conducted experiments in emulated field scenarios where the wireless IoT sensor nodes are likely to be deployed. The cases considered are: factory floor settings for hazardous chemical sensing, garden/agricultural field setting for water or pesticide level sensing, and roadside scenario for monitoring pollution level or recording different vehicular data.

In the experimental set-up (Figs. 9, 10 for estimating the $K$ parameter in different scenarios, the RF source (transmitter antenna) and the RF harvesting receiver node were placed on tripod stands with adjustable heights.

The other hardware unit specifications are as follows: 
TABLE IV: Energy saving using the proposed approach. Pol.: polarization; H: horizontal; V: vertical.

\begin{tabular}{|c|c|c|c|c|c|c|c|}
\hline$L(\mathrm{~m})$ & Pol. & $h_{\text {opt }}^{\text {Sim }}(\mathrm{cm})$ & $h_{\text {opt }}^{\text {Exp }}(\mathrm{cm})$ & $P_{\mathcal{H}}^{(\text {Friis })}(\mathrm{mW})$ & $P_{\mathcal{H}}^{(\text {prop })}(\mathrm{mW})$ & $P_{\mathcal{H}}^{(\text {Exp })}(\mathrm{mW})$ & $\begin{array}{c}\text { Energy saved (kJ): } \\
\text { numerical/experimental }\end{array}$ \\
\hline \multirow{2}{*}{0.8} & $\mathrm{~V}$ & 100 & 100 & 2.42 & 12.32 & 11.60 & $7.47 / 7.36$ \\
\cline { 2 - 8 } & $\mathrm{H}$ & 80.5 & 70 & 1.81 & 4.61 & 5.36 & $7.55 / 8.23$ \\
\hline \multirow{2}{*}{1.2} & $\mathrm{~V}$ & 100 & 100 & 1.1 & 5.88 & 5.05 & $16.62 / 15.99$ \\
\cline { 2 - 8 } & $\mathrm{H}$ & 15 & 15 & 1.03 & 2.43 & 3.05 & $12.58 / 14.47$ \\
\hline \multirow{2}{*}{1.5} & $\mathrm{~V}$ & 100 & 100 & 0.69 & 3.60 & 3.21 & $26.35 / 26.60$ \\
\cline { 2 - 8 } & $\mathrm{H}$ & 17.40 & 15 & 0.93 & 1.81 & 1.98 & $1.76 / 12.83$ \\
\hline \multirow{2}{*}{2.0} & $\mathrm{~V}$ & 98.30 & 90 & 0.37 & 2.21 & 1.94 & $1.62 / 49.21$ \\
\cline { 2 - 7 } & $\mathrm{H}$ & 22.10 & 15 & 0.77 & 1.21 & 1.45 & 13.70 \\
\hline
\end{tabular}

- $\boldsymbol{R F}$ source: Agilent RF synthesizer N9310A was used as $\mathrm{RF}$ source. It was set to transmit at a fixed frequency 915 $\mathrm{MHz}$ and fixed transmit power $10 \mathrm{dBm}$.

- Spectrum analyzer: Agilent Fieldfox Spectrum Analyzer N9918A was used to analyze the frequency and power profile of the received signal.

- Data acquisition and hardware control: The received power data was acquired using the trace function for different distances. Further the data was captured and automatically stored in a excel file.

- Antennas: In these experiments the same antennas [2] were used as in received power characterization experiments in Section IV] A directional antenna having a linear gain of $4.1(6.1 \mathrm{dBi})$ and half power beamwidth of $122^{\circ}$ (azimuth) and $68^{\circ}$ (elevation) was used at the transmitter, whereas an omnidirectional antenna with a linear gain of $1.25(1.0 \mathrm{dBi})$ was used at the receiver.

The experimental results on statistical channel parameters are discussed in Section VII-B

\section{ViI. Performance Results}

In this section first the numerical results of optimization algorithm proposed in Section $\mathrm{V}$ are discussed. Then, statistical channel model parameters estimated via experiments conducted for different scenarios in Section $\mathrm{VI}$ are presented.

\section{A. Optimum RF-ET Performance Gains}

Here, numerical results on achievable RF-ET performance with the proposed optimization Algorithm 1 is discussed.

1) Complexity versus Accuracy: First we discuss the optimum partitioning as complexity versus optimization accuracy tradeoff. Fig. 11 shows the variation of total number of computations against the desired level of accuracy $100 *(1-$ $\left.\frac{\epsilon}{h_{\max }-h_{\min }}\right)$. The plots clearly show that, for all tolerance values, complexity of the proposed algorithm with any number of partitions is less than that of brute force method.

To quantify the maximum number of partitions $m$ needed for achieving the same result as in brute force method, efficiency of the three competitive methods, namely, brute force, golden search with variable partitions, and Friis equation (1), are plotted in Fig. 12 against transmitter-receiver distance with receiver antenna height $0.8 \mathrm{~m}$. It is noted that, optimized efficiency is obtained with 3 partitions at all transmitter-receiver distance and with $\epsilon=0.001$ for metallic reflecting ground surface. The main reason of 3 partitions being sufficient is

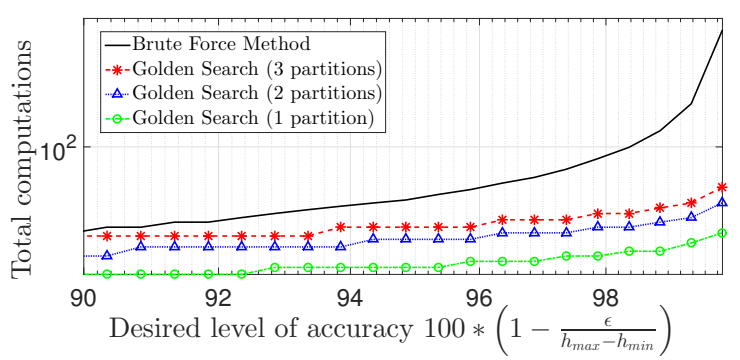

Fig. 11: Total computations versus level of accuracy.

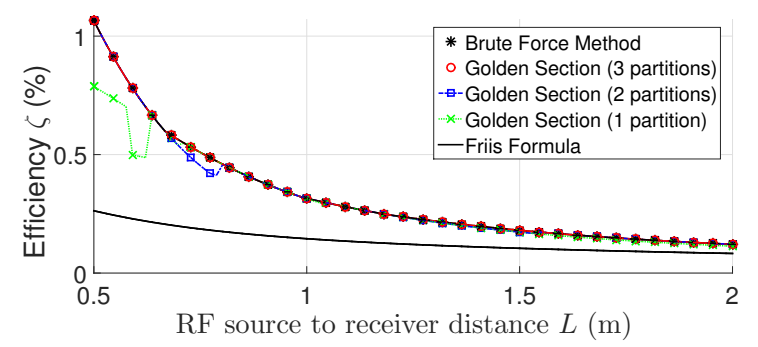

Fig. 12: Efficiency comparison for different number of partitions.

that the RF-ET range is very limited due to path loss, low RF harvesting sensitivity, low power harvester circuit efficiency, and limited transmit power. The other intuition behind choosing only 3 partitions is that, for the considered different parameters values, the phase difference between the LOS and NLOS component is always less than $6 \pi$. Therefore, only 3 different constructive and destructive regions are formed, as also confirmed from the received power expression in (8).

It can also be observed that, at all distances and for all values of partitions, the proposed algorithm offers much better RFET performance compared to the estimate using Friis formula. With Powercast P1110 energy harvesting module (used in our experiments), the received power must be more than $-5 \mathrm{dBm}$ for charging of the supercapacitor at a reasonably fast rate [2]. This practically limits the range of RF-ET to a few meters only, and 3 partitions are sufficient to make the problem unimodal in each partition.

2) Experimental Verification and RF-ET Performance Comparison: To verify the numerical results of the proposed optimization algorithm, we have conducted further end-to-end hardware experiments with the metallic ground reflector. The experimental devices used are as described in Section VIC. An additional power amplifier has been used to match 1 Watt transmit power as considered in simulation. The used mini-circuit ZHL-42W+ power amplifier was first calibrated 


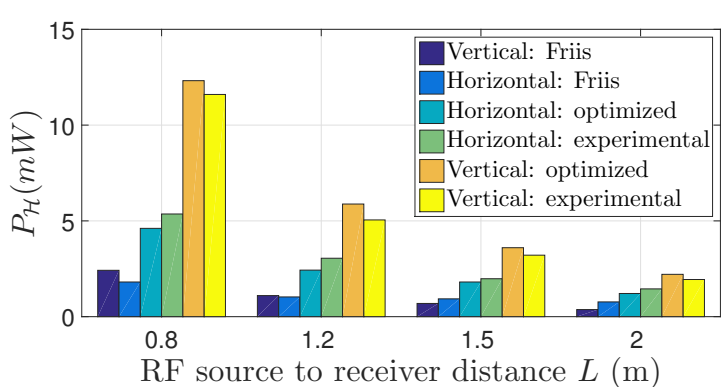

Fig. 13: Optimum efficiency comparison and verification with endto-end hardware experiments.

before usage. The amplifier gain obtained is $26.1 \mathrm{~dB}$ for a $\mathrm{dc}$ input of $15 \mathrm{~V}$. Powercast P1110 RF energy harvester has been used to quantify the harvested power at receiver node [2]. The experimental results along with simulated ones are as shown in Table IV. The normalized RMSE of numericallycomputed optimum received power values with respect to the experimentally-obtained results are up to 0.0577 , which is within the tolerable range as noted in [22] - thus corroborating the analytical optimization.

To demonstrate gain from the proposed model with the optimized antenna heights, the optimum harvested power is plotted in Fig. 13. for both horizontal and vertical polarization. These results were obtained with metallic ground reflector where transmitting and receiving antenna were respectively directional and omnidirectional, and transmitter height is varied from 0.15 to $1 \mathrm{~m}$. The experimental results on optimum transmit antenna height, received power, and harvested power match well with the numerical results, thus validating the numerical optimization. Further, from the plots it is apparent that harvested energy with the proposed model is always higher than that with Friis equation. The difference between harvested power with horizontal and vertical polarization is due to different gain of transmitting antenna, and also the reflection coefficient is different for the two polarizations.

3) Energy Saving: To calculate the energy saving by optimally placing nodes, we first need to find the time required to charge a supercapacitor $C$ to a certain voltage $V$ with a constant received power $P_{\mathcal{H}}$. Referring to Fig 7 (a) we have:

$$
P_{\mathcal{H}}=V(t) I(t)=\frac{q}{C} \frac{d q}{d t} \rightarrow \int_{0}^{Q} q d q=\int_{0}^{t} P_{\mathcal{H}} C d t
$$

Solving (24), the time required to charge $C$ to $V=Q / C$ is $T=\frac{C V^{2}}{2 P_{\mathcal{H}}}$. Higher harvested power saves crucial system time and hence the energy consumed at the transmitter. To numerically quantify the energy saving, we have considered Agilent N9310A RF synthesizer as the RF source which consumes up to $100 \mathrm{~W}$ [27] while it transmits. With Powercast P1110 having $C=50 \mathrm{mF}$ and terminal charging voltage $V=3$ volts, energy saved for different transmitter-receiver distance $L$ are noted in Table IV Similarly, the increase in received power with optimum transmitter antenna height can be translated into extension in the RF-ET range.

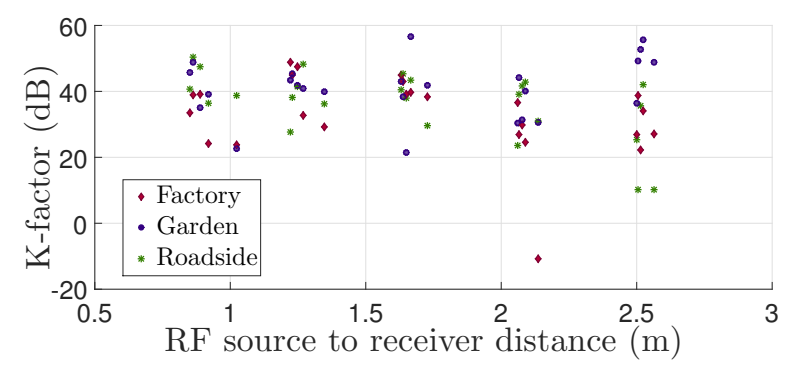

Fig. 14: K-factor versus end-to-end distance: vertical polarization.

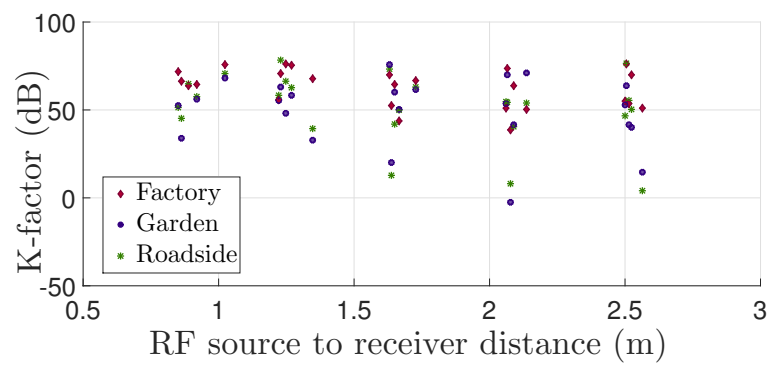

Fig. 15: K-factor versus end-to-end distance: horizontal polarization.

TABLE V: Mean $K$-factor in different scenarios. H: horizontal polarization, V: vertical polarization.

\begin{tabular}{|c|c|c|c|c|c|c|}
\hline Scenarios & \multicolumn{2}{|c|}{ Garden } & \multicolumn{2}{c|}{ Roadside } & \multicolumn{2}{c|}{ Factory floor } \\
\hline \multirow{2}{*}{$K(\mathbf{d B})$} & $\mathrm{H}$ & $\mathrm{V}$ & $\mathrm{H}$ & $\mathrm{V}$ & $\mathrm{H}$ & $\mathrm{V}$ \\
\cline { 2 - 7 } & 47.309 & 40.938 & 51.232 & 36.152 & 62.742 & 32.942 \\
\hline
\end{tabular}

\section{B. Experimental Estimates on Statistical Channel Parameters}

We now discuss quantitative estimates of the statistical channel parameters for RF-ET based on the models presented in the Section VI.

1) Estimation of Rician K-Factor: The experiments in Section VI-C were conducted for all the three different scenarios and the Rician $K$-factor calculated from the received power values are plotted in Figs. 14 and 15 for horizontal and vertical polarization, respectively. From the plots it can be inferred that the $K$-factor is very high for all possible combinations except for a few outlier data points. This observation affirms that Rayleigh distribution is not a good model for characterizing the fading in RF-ET. Mean $K$ values are also listed in Table $\mathrm{V}$ for all three deployment scenarios.

2) Estimation of Path Loss and Shadow Fading Parameters: Both $A$ and $\gamma$ in (23) are evaluated by fitting a liner curve through all the received power data points so as to minimize the root mean square error (RMSE). For the factory floor setting, this is shown on a log-log plot in Fig. 16. Similar results were obtained in the other two deployment scenarios. The shadow fading term is a zero mean $(\mu=0)$ Gaussian random variable where the only unknown is the variance $\varsigma$. In the three experiments we have taken $z_{o}$ to be $75 \mathrm{~cm}$. The different parameter values estimated are as listed in Table VI As a general trend, it can be observed that the constant $A$ (defined in (23)) is smaller for horizontal polarization than that for vertical polarization. The path-loss exponent is more than 2 for all the different scenarios, however, it can be clearly 


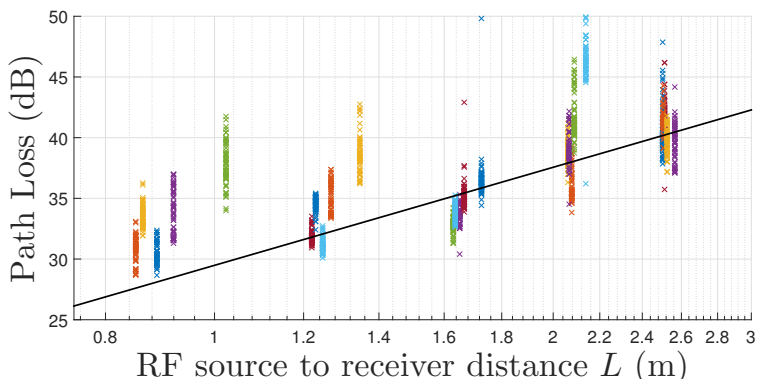

Fig. 16: Path loss versus distance scatter plot in factory floor setting.

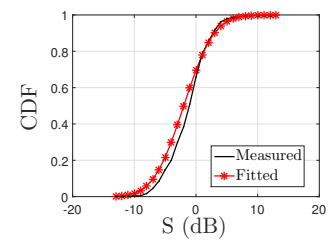

(a) Roadside

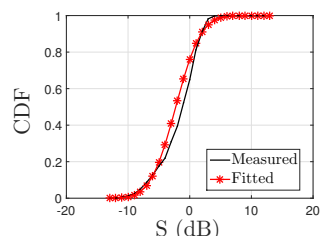

(b) Factory floor
Fig. 17: CDF comparison of the fitted and measured data for shadow fading component.

TABLE VI: Numerical values for different parameters used in statistical path loss modeling.

\begin{tabular}{|c|c|c|c|c|c|}
\hline Scenarios & Polarization & $A(\mathrm{~dB})$ & $\gamma$ & $\varsigma(\mathrm{dB})$ & RMSE \\
\hline \multirow{2}{*}{ Roadside } & Horizontal & 17.9986 & 2.4826 & 2.9160 & .0406 \\
\cline { 2 - 6 } & Vertical & 23.6306 & 2.0928 & 3.8707 & .0166 \\
\hline \multirow{2}{*}{ Garden } & Horizontal & 18.8362 & 2.1974 & 3.3164 & .0301 \\
\cline { 2 - 6 } & Vertical & 24.1659 & 2.2085 & 3.1692 & .0365 \\
\hline \multirow{2}{*}{ Factory } & Horizontal & 19.1599 & 2.2232 & 2.6586 & 0.0237 \\
\cline { 2 - 6 } & Vertical & 26.1294 & 2.6833 & 3.2057 & .0235 \\
\hline
\end{tabular}

observed that, it is much less than commonly used value of 4 as in [19]. The RMSE in all the scenarios is well within the acceptable bound of 0.08 [22]. Fig. 17] shows a good match between the CDF drawn from the measured values and the Gaussian fitted curve, confirming the log-normal shadow fading component.

\section{CONCLUdING REMARKS}

In this paper, first we have presented a new deterministic channel model for accurate characterization of received power via RF-ET at an energy harvesting IoT field node. In determining received power, the proposed model incorporates the effect of NLOS component along with the other important factors, namely, polarization mismatch, antenna gain patterns, and RF harvesting efficiency, which were not considered before. Results from extensive experiments in a controlled environment (anechoic chamber) have validated the model. The proposed model has been further used to maximize the utility of NLOS component in enhancing the RF-ET efficiency, wherein through an optimization formulation optimum transmitter antenna height has been obtained for a given receiver distance and antenna height. To solve this nonconvex optimization problem, a simple global optimization algorithm based on golden section method has been proposed which converges logarithmically to the optimal solution.
Noting that, in practical deployments the IoT nodes are expected to experience stochastic environment, we have modeled the RF-ET channel as Rician distributed along with path loss and log-normal shadow fading. Via extensive experiments we have characterized the wireless channel parameters in three typical deployment scenarios: factory floor, garden/agricultural field, and roadside/marketplace installations.

Numerical results show that the proposed optimized model offers up to three times higher RF-ET efficiency as compared to the standard Friis formula based estimate. Further, the experimental studies have quantified the statistical channel parameter values in emulated practical settings, which also clearly assert that Rayleigh fading is not well-suited for RF-ET analysis. The developed model and the experimental findings are expected to play very useful roles in analyzing the performance of RF harvesting wireless networks.

\section{REFERENCES}

[1] S. Kumar, D. Mishra, and S. De, "An accurate channel model for optimizing effect on non-LOS component in RF energy transfer," in Proc. Nat. Conf. Commun., Chennai, India, Mar. 2017, pp. 1-6.

[2] Powercast. [Online]. Available: http://www.powercastco.com.

[3] P. Nintanavongsa, U. Muncuk, D. R. Lewis, and K. R. Chowdhury, "Design optimization and implementation for RF energy harvesting circuits," IEEE J. Emerging and Sel. Topics in Circuits Syst., vol. 2, no. 1, pp. 24-33, Mar. 2012.

[4] D. Mishra and S. De, "Optimal relay placement in two-hop RF energy transfer," IEEE Trans. Commun., vol. 63, no. 5, pp. 1635-1647, May 2015.

[5] H. Tabassum, E. Hossain, A. Ogundipe, and D. I. Kim, "Wirelesspowered cellular networks: Key challenges and solution techniques," IEEE Commun. Mag., vol. 53, no. 6, pp. 63-71, June 2015.

[6] I. Flint, X. Lu, N. Privault, D. Niyato, and P. Wang, "Performance analysis of ambient RF energy harvesting with repulsive point process modeling," IEEE Trans. Wireless Commun., vol. 14, no. 10, pp. 54025416, Oct. 2015.

[7] T. K. Sarkar, Z. Ji, K. Kim, A. Medouri, and M. Salazar-Palma, "A survey of various propagation models for mobile communication," IEEE Antennas propag. Mag., vol. 45, no. 3, pp. 51-82, June 2003.

[8] M. J. Feuerstein, K. L. Blackard, T. S. Rappaport, S. Y. Seidel, and H. H. Xia, "Path loss, delay spread, and outage models as functions of antenna height for microcellular system design," IEEE Trans. Veh. Technol., vol. 43, no. 3, pp. 487-498, Aug. 1994.

[9] M. Y. Naderi, K. R. Chowdhury, and S. Basagni, "Wireless sensor networks with RF energy harvesting: Energy models and analysis," in Proc. IEEE Wireless Commun. Netw. Conf., New Orleans, USA, Mar. 2015, pp. 1494-1499.

[10] D. Mishra, S. De, and C. F. Chiasserini, "Joint optimization schemes for cooperative wireless information and power transfer over Rician channels," IEEE Trans. Commun., vol. 64, no. 2, pp. 554-571, Feb. 2016.

[11] D. Mishra, S. De, and D. Krishnaswamy, "Dilemma at RF energy harvesting relay: Downlink energy relaying or uplink information transfer?" IEEE Trans. Wireless Commun., vol. 16, no. 8, pp. 4939-4955, Aug. 2017.

[12] L. J. Greenstein, S. S. Ghassemzadeh, V. Erceg, and D. G. Michelson, "Ricean $K$-factors in narrow-band fixed wireless channels: Theory, experiments, and statistical models," IEEE Trans. Veh. Technol., vol. 58, no. 8, pp. 4000-4012, Oct. 2009.

[13] V. Erceg, L. J. Greenstein, S. Y. Tjandra, S. R. Parkoff, A. Gupta, B. Kulic, A. A. Julius, and R. Bianchi, "An empirically based path loss model for wireless channels in suburban environments," IEEE $J$. Sel. Areas Commun., vol. 17, no. 7, pp. 1205-1211, July 1999.

[14] S. De and R. Singhal, "Toward uninterrupted operation of wireless sensor networks," IEEE Computer Mag., vol. 45, no. 9, pp. 24-30, Sept. 2012.

[15] S. Bi, Y. Zeng, and R. Zhang, "Wireless powered communication networks: An overview," IEEE Wireless Commun., vol. 23, no. 2, pp. 10-18, Apr. 2016. 
[16] I. Krikidis, S. Timotheou, S. Nikolaou, G. Zheng, D. W. K. Ng, and R. Schober, "Simultaneous wireless information and power transfer in modern communication systems," IEEE Commun. Mag., vol. 52, no. 11, pp. 104-110, Nov. 2014.

[17] D. Mishra, S. De, S. Jana, S. Basagni, K. Chowdhury, and W. Heinzelman, "Smart RF energy harvesting communications: challenges and opportunities," IEEE Commun. Mag., vol. 53, no. 4, pp. 70-78, Apr. 2015.

[18] C. A. Balanis, Antenna Theory: Analysis and Design. John Wiley \& Sons, 2012.

[19] T. S. Rappaport, Wireless Communications: Principles and Practice. Prentice Hall PTR New Jersey, 1996.

[20] D. J. Griffiths, Introduction to electrodynamics. Pearson Education Inc., 2005.

[21] Ets Lindgren. Horn antenna Model no. 3115 Manual. [Online]. Available: http://www.ets-lindgren.com/manuals/3115.pdf

[22] D. Hooper, J. Coughlan, and M. R. Mullen, "Structural equation modeling: Guidelines for determining model fit," Electronic J. Business Research Methods, vol. 6, no. 1, Apr. 2008.

[23] E. W. Weisstein. Least squares fitting. Wolfram MathWorld: A Web Resource, Champaign, IL, USA. Accessed on 8th July, 2017. [Online]. Available: http://mathworld.wolfram.com/LeastSquaresFitting.html

[24] M. K. Simon and M.-S. Alouini, Digital communication over fading channels. John Wiley \& Sons, 2005.

[25] L. J. Greenstein, D. G. Michelson, and V. Erceg, "Moment-method estimation of the Ricean $K$-factor," IEEE Commun. Lett., vol. 3, no. 6, pp. 175-176, June 1999.

[26] A. Goldsmith, Wireless communications. Cambridge university press, 2005.

[27] Agilent N9310A RF signal generator. [Online]. Available: http: //www.keysight.com/upload/cmc_upload/All/N9310AUsersGuide.pdf

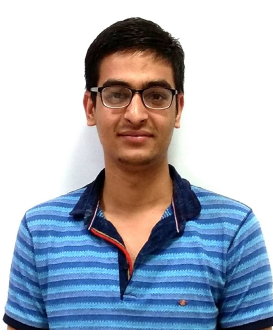

Sidharth Kumar received the B.Tech. degree in electrical engineering from the Indian Institute of Technology, Delhi, in 2017, where he is currently working toward the M.S.(Research) degree. His research interests include wireless energy transfer, computer networks, machine learning, aerial communication systems and internet of things (IoT).

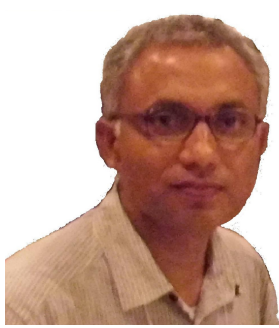

Swades De (S'02-M'04-SM'14) received his B.Tech. degree in Radiophysics and Electronics from the University of Calcutta in 1993, the M.Tech. degree in Optoelectronics and Optical communication from IIT Delhi in 1998, and the Ph.D. degree in Electrical Engineering from the State University of New York at Buffalo in 2004.

He is currently a Professor with the Department of Electrical Engineering, IIT Delhi. Before moving to IIT Delhi in 2007, he was a Tenure-Track Assistant Professor with the Department of ECE, New Jersey Institute of Technology, Newark, NJ, USA, from 20042007. He worked as an ERCIM Post-doctoral Researcher at ISTI-CNR, Pisa, Italy (2004), and has nearly five years of industry experience in India on telecom hardware and software development, from 19931997, 1999. His research interests are broadly in communication networks, with emphasis on performance modeling and analysis. Current directions include energy harvesting sensor networks, broadband wireless access and routing, cognitive/white-space access networks, smart grid networks, and IoT communications. Dr. De currently serves as a Senior Editor of IEEE COMMUNICATIONS LETTERS, and an Associate Editor of IEEE TRANSACTIONS ON VEHICULAR TECHNOLOGY, IEEE WIRELESS COMMUNICATIONS LETTERS, Springer Photonic Network Communications, and the IETE Technical Review Journal.

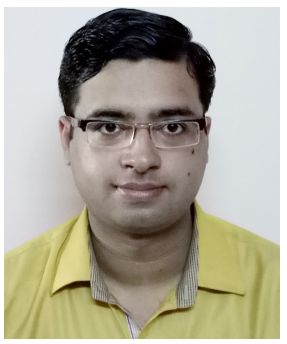

Deepak Mishra (S'13-M'17) received the B.Tech degree in Electronics and Communication Engineering from the Guru Gobind Singh Indraprastha University, Delhi, India, in 2012, and the Ph.D. degree in Electrical Engineering from the Indian Institute of Technology Delhi, India, in 2017. He is currently a Postdoctoral Researcher in the Department of Electrical Engineering (ISY), Linköping University, Linköping, Sweden. His research interests include energy harvesting cooperative communication networks, massive MIMO, physical layer security, signal processing and energy optimization schemes for uninterrupted operation of wireless networks.

Dr. Mishra was selected as an Exemplary Reviewer of the IEEE TRANSACTIONS ON WIRELESS COMMUNICATIONS for 2017. He was a recipient of IBM Ph.D. Fellowship, Raman-Charpak Fellowship, and Endeavour Research Fellowship awards in 2016, 2017, and 2018, respectively. 